Missed Diagnosis

\title{
Abdominal epilepsy misdiagnosed as psychogenic pain
}

\author{
Pratibha D. Singhi ${ }^{1}$ and Sanjam Kaur ${ }^{2}$ \\ Departments of ${ }^{1}$ Pediatrics and ${ }^{2}$ Psychiatry, Postgraduate Institute of Medical Education and Research, \\ Chandigarh 160 012, India.
}

\begin{abstract}
Summary: The authors present the case report of a child with abdominal epilepsy who had suffered from abdominal pain for several months under the label of psychogenic pain. The important historical clues were pallor and cold sweating during the paroxysm, followed by lethargy and prolonged sleep. An abnormal electroencephalogram and a remarkable response to anticonvulsants confirmed the diagnosis. This condition must be considered in a child with undiagnosed recurrent abdominal pain.
\end{abstract}

\section{Introduction}

Recurrent abdominal pain is a common problem in children. Sometimes, in spite of multiple diagnostic tests, no cause can be found. These children may therefore be labelled as having 'psychogenic pain'. Abdominal epilepsy is a rare but easily treatable cause of recurrent paroxysmal abdominal pain. In spite of repeated reminders ${ }^{1-3}$ it continues to escape the attention of paediatricians. The case report of a child with abdominal epilepsy who continued to suffer from abdominal pain for several months under the label of 'psychogenic pain' is presented to remind practitioners about this unusual but easily treatable condition.

\section{Case report}

A 10 year old boy was brought one year ago to the paediatric outpatient clinic with a history of recurrent, paroxysmal abdominal pain for 3 years. To begin with, he had attacks of pain once in 2-3 months for a few minutes. Later, the frequency, intensity and duration increased progressively and for the past 6 months he was having several attacks every week; his physical examination and a number of investigations including repeated stool and urine examinations, blood counts, blood chemistry, and barium studies were normal. He did not respond to analgesics, antispasmodics, and antihelminthics. On the contrary, his pain kept increasing in frequency

Correspondence: P.D. Singhi, M.D.

Accepted: 3 September 1987 and severity. He was thought to have functional pain and was referred to the child guidance clinic. Here he was examined by two psychiatrists and labelled as having an hysterical conversion disorder. At this stage, he was being considered for inclusion in a study of psychogenic pain, and was brought to us for paediatric evaluation. A careful review of the history revealed that the child had paroxysms of severe periumbilical pain during which he became pale and was cold to the touch. He became lethargic and went off to sleep after these attacks. There were no convulsions. Also, no undue stress factors or secondary gains could be elicited from the history. A diagnosis of abdominal epilepsy was therefore suggested, and an electroencephalogram (EEG) done. This showed a background rhythm of 8-9 Hz and $15-30 \mathrm{mw}$ along with fast beta activity. Sharp spikes, and spike and wave activity arising over the central region and becoming generalized, indicating epilepsy, were seen (Figure 1). The child was put on phenobarbitone to which he showed a remarkable response. However, after a month, he developed hyperkinetic behaviour and hence this was replaced by phenytoin. He continued to improve and has been asymptomatic for the past 6 months.

\section{Discussion}

This case illustrates the problem of a child with recurrent paroxysmal abdominal pain who remained undiagnosed and continued to suffer for

(C) The Fellowship of Postgraduate Medicine, 1988 


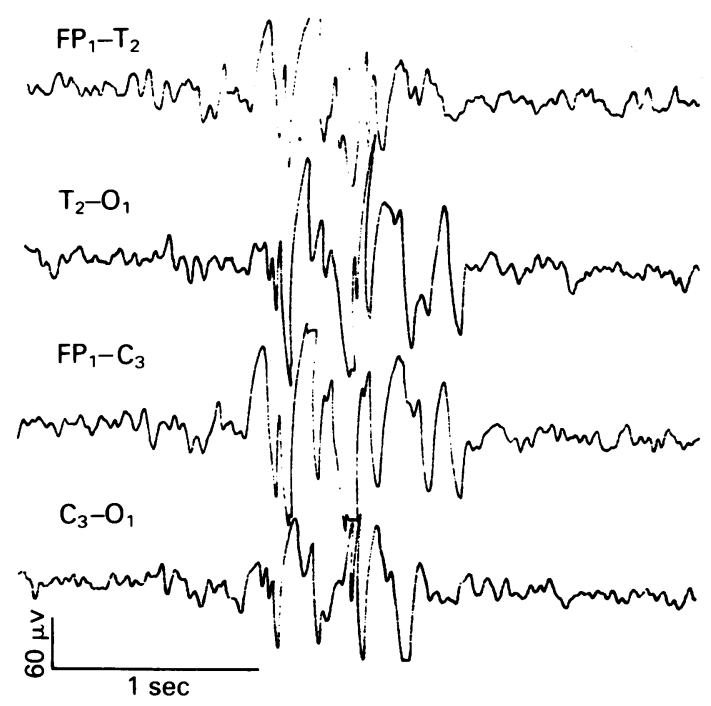

Figure 1 EEG recording done in the awake state with electrode placement as indicated by standard abbreviations. Background rhythm shows mixed $7 \mathrm{~Hz}$ and fast beta activity. There is bilateral discharge of polyspikes and waves and spikes and waves.

several months under the label of psychogenic pain. It was only on careful review of the history that the diagnosis of abdominal epilepsy was made. The main clues in the history were (a) alteration of awareness, as indicated by lethargy and prolonged

\section{References}

1. Schade, G.H. \& Gofman, H. Abdominal epilepsy in childhood. Pediatrics 1960, 25: 151-154.

2. Douglas, E.F. \& White, P.T. Abdominal epilepsy - a reappraisal. J Pediatr 1971, 78: 59-67.

3. Zarling, E.J. Abdominal epilepsy: An unusual cause of sleep after the attack, (b) autonomic phenomena in the form of pallor and cold-sweating during the attack. The EEG showing definite evidence of epilepsy, and the remarkable response to anticonvulsants, confirmed the diagnosis.

Recurrent abdominal pain may often become a diagnostic problem in children. The importance of a careful history and examination which may give useful clues towards aetiology cannot be overemphasized. Abdominal epilepsy is an easily treatable cause of paroxysmal abdominal pain. The criteria laid down for its diagnosis ${ }^{2}$ are (i) paroxysmal abdominal pain, (ii) exclusion of abdominal visceral pathology, (iii) alteration of mental status during some of the attacks, (iv) definitely abnormal electro-encephalographic tracing and (v) response to anticonvulsant therapy. Not all the criteria need be present in each case. This child had all the criteria.

A similar picture may also be seen in abdominal migraine. However, in this condition, the pain is usually gradual in onset, a family history of migraine is often present, and the EEG is normal or shows only minor abnormalities. ${ }^{4}$ Stress induced abdominal pain can also be differentiated by the elucidation of stress factors, and by a normal EEG.

To conclude therefore, a diagnosis of abdominal epilepsy must be considered in children with recurrent paroxysmal abdominal pain, especially in those with suggestive historical data, and an EEG should be done. A vigilant approach can save a child from a lot of unwarranted suffering.

recurrent abdominal pain. Am J Gastroenterol 1984, 79: 687-688.

4. Prichard, J.S. Abdominal pain of cerebral origin in children. Can Med Assoc J 1958, 28: 665-667. 